\title{
Ovarian Dysgerminoma in Pregnant Women with Viable Fetus: A Rare Case Report
}

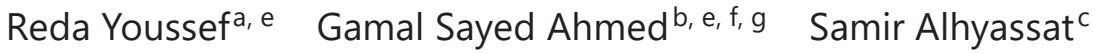 \\ Sanaa Badra Ahmed Sabry ${ }^{a}$ e Samah Kohlad, e \\ aDepartment of Clinical Imaging, Women's Wellness and Research Center, Hamad Medical \\ Corporation, Doha, Qatar; ${ }^{b}$ Department of Obstetrics and Gynecology, Women's Wellness \\ and Research Center, Hamad Medical Corporation, Doha, Qatar; ${ }^{\mathrm{c}}$ Anatomic Pathology \\ Division, Department of Laboratory Medicine and Pathology, Hamad Medical Corporation, \\ Doha, Qatar; ${ }^{d}$ Hematopathology Division, Department of Laboratory Medicine and

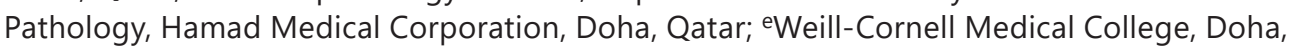 \\ Qatar; ${ }^{f}$ Clinical Department, College of Medicine, QU Health, Qatar University, Doha, Qatar; \\ gUniversity of Dundee, Dundee, UK
}

\section{Keywords}

Ovarian dysgerminoma - Feto-maternal compromise - Dysgerminoma - Dysgerminoma in pregnancy - Ovarian malignancy · Malignancy in pregnancy

\begin{abstract}
Dysgerminoma is an uncommon malignant tumor arising from the germ cells of the ovary. Its association with pregnancy is extremely rare, with a reported incidence of about $0.2-1$ per 100,000 pregnancies. Women in the reproductive age group are more commonly affected. It can be extremely rare to conceive naturally, without assisted reproductive interventions, in cases with ovarian dysgerminoma. If a pregnancy does occur with a concurrent dysgerminoma, it is even more unusual to carry the pregnancy to viability or childbirth without fetal or maternal compromise. We report a case of right ovarian dysgerminoma in a young female with a viable intrauterine pregnancy at 10 weeks, which is rarely diagnosed and managed at this gestational age. Numerous factors played a role in her favorable outcome, including early suspicion by ultrasound and presenting history, surgery, histopathological assessment, imaging, and involvement of the multidisciplinary oncology team. Ovarian neoplasms may rapidly increase in size within a short period with little or no symptoms. This poses a diagnostic challenge for obstetricians and oncologists. Hence, we aimed to evaluate the role of imaging in pregnancy using ultrasound as an imaging modality for both early detection of ovarian neoplasms and for follow-up. In conclusion, patients with ovarian dysgerminoma in pregnancy can have favorable outcomes. Treatment should be individualized on a case-to-case basis,
\end{abstract}

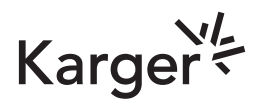


depending on many factors; cancer stage, previous reproductive history, the impact of imaging in staging or follow-up of tumor on the fetus, fetal gestational age, and whether termination of the pregnancy can improve survival or morbidity for the mother.

(C) 2021 The Author(s).

Published by S. Karger AG, Basel

\section{Introduction}

Dysgerminomas are germ cell ovarian tumors. About $20 \%$ of all ovarian tumors originate from germ cells whereas only $3 \%$ of them are malignant. Dysgerminomas account for about $1 \%$ of all germ cell tumors, but they are frequently malignant [1]. They affect young females, prevalently during childhood, and the vast majority of them need and respond well only to chemotherapy. The problem arises when dysgerminoma is diagnosed in women of reproductive age who have never given birth and require a surgical procedure. A more serious situation occurs if dysgerminomas develop in young patients during pregnancy, which poses various medical and ethical dilemmas.

\section{Case Report}

A 36-year-old patient, gravida 7 para 2, pregnant 10 weeks and 3 days, presented to our emergency department complaining of severe right-sided lower abdominal pain and dizziness. She had previously had two normal vaginal deliveries. In the emergency department, the pain was on and off, with no chills or fever, no associated weight loss, and no bowel or urinary symptoms. Prior to this pregnancy, there had been no menstrual irregularities and she had not been on any form of contraception.

On physical examination, the patient had normal vital signs. The chest was clear with no added cardiovascular sounds. The abdomen was soft with no signs of peritonism, with moderate to marked tenderness in the right iliac fossa. Pelvic ultrasound revealed a 10 weeks and 3 days viable fetus with an enlarged right ovary measuring $7.5 \times 4.5 \mathrm{~cm}$ in abnormal location in the right lower abdomen. A large area of the right ovary was seen occupied by a heterogeneous pattern (Fig. 1), but with a normal-appearing Doppler study (dual blood supply). The left ovary appeared unremarkable, measuring $3.8 \times 2.0 \mathrm{~cm}$.

The ultrasound findings collectively raised the probability of right ovarian torsion, but an underlying right ovarian lesion could not be excluded; it was suggested to correlate the ultrasound findings with the clinical picture. Based on the clinical and ultrasonographic findings of torsion, the patient consented to an urgent laparoscopy/laparotomy with ovarian cystectomy and oophorectomy.

Fig. 1. Ultrasound images showing a viable intrauterine fetus of 10 weeks and 3 days with associated enlarged right ovary seen occupied by heterogeneous pattern (red arrow), features suggesting right ovarian torsion with suspecious underlying right ovarian lesion.
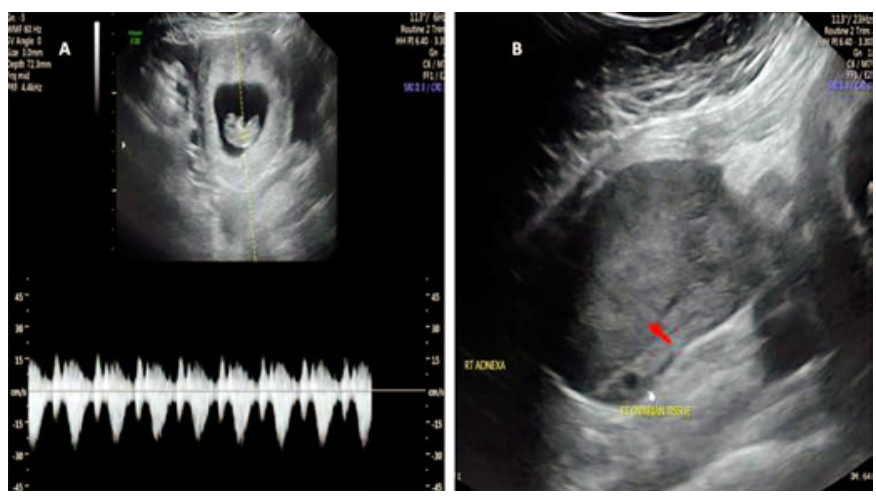


\section{Case Reports in Oncology}

\begin{tabular}{l|l}
\hline Case Rep Oncol 2021;14:141-146 \\
\hline DOI: 10.1159/000513622 & $\begin{array}{l}\text { ○ 2021 The Author(s). Published by S. Karger AG, Basel } \\
\text { www.karger.com/cro }\end{array}$ \\
\hline
\end{tabular}

Youssef et al.: Ovarian Dysgerminoma in Pregnant Women with Viable Fetus

Fig. 2. Intraoperative view of the twisted suspicious right ovary $(8.0 \times 5.0 \mathrm{~cm})$.

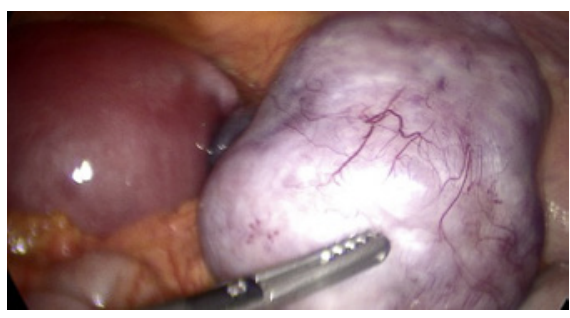

Fig. 3. Background lymphocytes surrounding sheets of tumor cells (H\&E, $\times 40$ ).

Fig. 4. Tumor cells with large clear cytoplasm $(H \& E, \times 200)$.
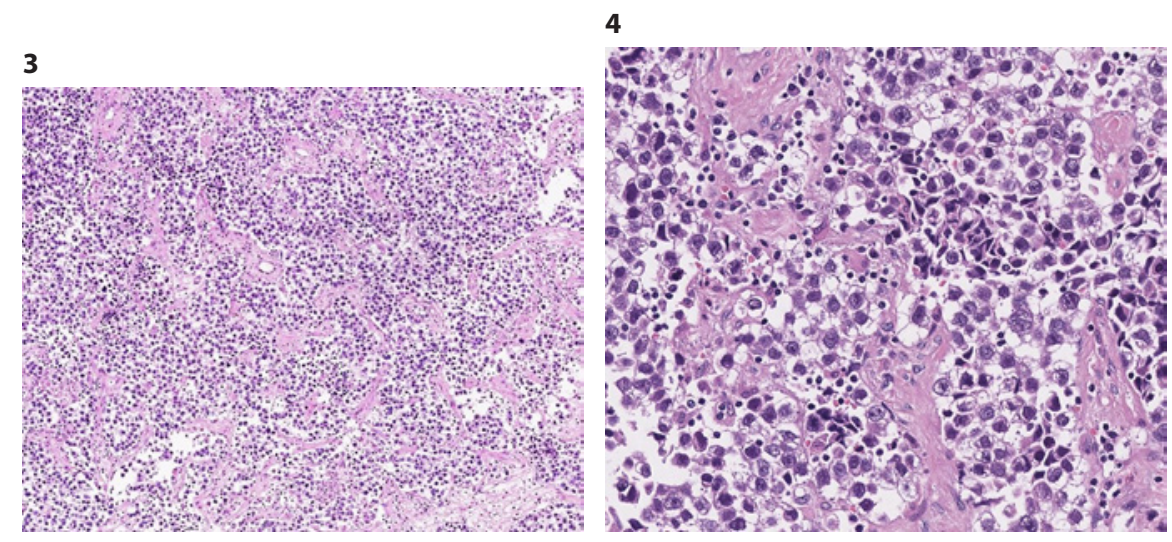

Intraoperatively, the right ovary was enlarged, appearing suspicious with a bosselated outer surface, and an intact capsule was noted (Fig. 2). No omental or peritoneal nodules, smooth liver surface, and undersurface of the diaphragm were seen. No abnormality was observed in any other intra-abdominal organs. The other ovary looked normal. A detorsion was performed and a decision was made to perform laparoscopic right oophorectomy. The specimen of the right ovary hosting the mass was sent to histopathology. Postoperatively, the patient made an uneventful recovery and was sent home the next day after debriefing her and the family and confirming the viability of the baby by ultrasound scan.

\section{Histopathological Findings}

Histology revealed an ovarian tumor (dysgerminoma).

Gross: The specimen consisted of an oophorectomy measuring $8.7 \times 5.5 \times 4.6 \mathrm{~cm}$ and weighing $101 \mathrm{~g}$. The outer surface was tan pink and focally hemorrhagic. A focal defect $3 \mathrm{~cm}$ in maximum diameter was noted, probably related to the intraoperative cyst exploration attempt. The outer surface was inked green. The specimen was serially sectioned to reveal homogeneous, tan white, solid, focally hemorrhagic cut surfaces. Representative sections were submitted in 21 cassettes.

Microscopic: The sections showed sheets and nests of large, uniform polygonal cells with clear and occasionally eosinophilic cytoplasm and distinct membranes. A background of lymphocytic infiltrate and thin branching blood vessels was noted. There was no evidence of vascular invasion, necrosis, or extension outside the testicular parenchyma. The immunohistochemical stains showed that the the tumor cells were positive for CD117 and OCT3/4. The immunohistochemical stains for beta-hCG, EMA, CD30, and alpha-fetoprotein were negative (Figure 3-6).

A postoperative ultrasound was performed and revealed a viable early pregnancy of $12+$ weeks with postsurgical changes in the right adnexa, yet no evidence of hematoma or 


\section{Case Reports in Oncology}

\begin{tabular}{l|l}
\hline Case Rep Oncol 2021;14:141-146 \\
\hline DOI: 10.1159/000513622 & $\begin{array}{l}\text { @ 2 2021 The Author(s). Published by S. Karger AG, Basel } \\
\text { www.karger.com/cro }\end{array}$ \\
\hline
\end{tabular}

5

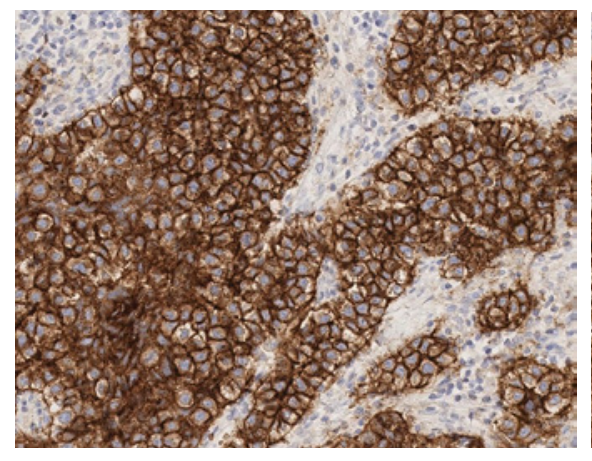

6

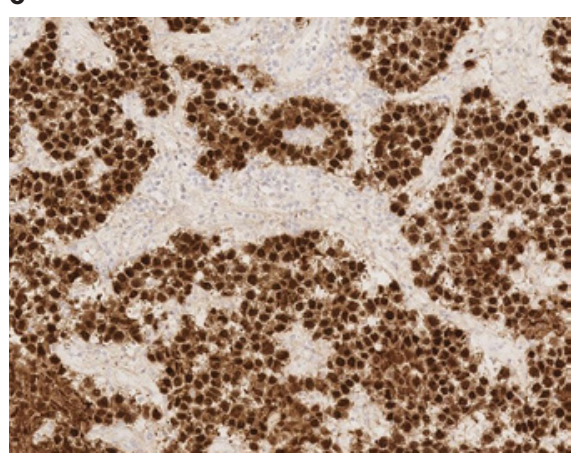

Fig. 5. Immunohistochemistry for CD117.

Fig. 6. Immunohistochemistry for OCT3/4.

Fig. 7. MRI. Coronal T2 and axial T2 FS weighted images showing evidence of right oophorectomy with postoperative changes in the form of a trace of free fluid in the right adnexa with no evidence of residual disease.
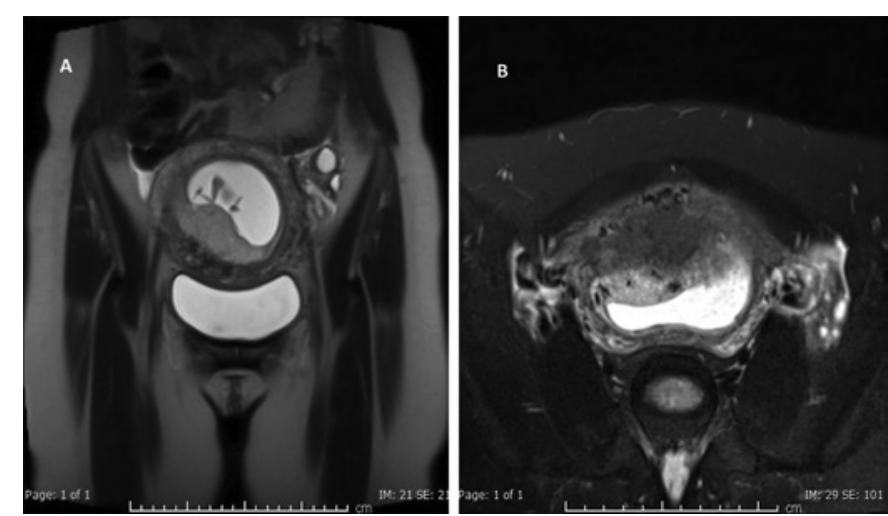

collection at the operative bed. A computed tomography (CT) scan of the chest was also performed, revealing no evidence of chest metastasis. Magnetic resonance imaging (MRI) of the abdomen and pelvis was unremarkable (Fig. 7).

The multidisciplinary team meeting considered all the factors, explored patient wishes, and a decision was made after CT of the chest and MRI of the abdomen to follow up the patient, allowing the pregnancy to continue.

\section{Discussion}

Dysgerminomas account for 1-5\% of all ovarian malignancies in the first two decades of life [2, 3]. Approximately $80 \%$ of cases are reported in patients aged $<30$ years (mean 21 years) [4].

Germ cell tumors can be curable, and the reproductive potential for these young women retained, after fertility-preserving surgery and chemotherapy for ovarian dysgerminoma. Most women had recovery of their menses within 9 months and many got pregnant after that $[5,6]$. Natural conception is also possible in these women [7]. However, the incidence of ovarian dysgerminoma in pregnancy is extremely rare at approximately $0.2-1$ per 100,000 pregnancies [8].

This poses a challenge for the treating physicians in both the diagnosis and the later management of confirmed cases. Few cases (4.7\%) of ovarian tumors that were surgically resected turned out to be malignant on subsequent histology. Even fewer $(0.9 \%)$ were confirmed by histopathology to be a dysgerminoma [9]. Indeed, with such an extremely 
rare clinical condition, misdiagnosis during pregnancy is not uncommon. A literature review revealed that it is not unusual to misdiagnose dysgerminoma by ultrasound examination and diagnose uterine fibroids instead. MRI has a sensitivity of about $98 \%$ for detecting the origin of an ovarian tumor. However, there have been reports of mistaking a malignant ovarian tumor for a pedunculated uterine fibroid with areas of cystic degeneration [10].

Ovarian tumors generally remain asymptomatic until they are discovered due to their large size or related complications [6]. In the current case, dysgerminoma was diagnosed due to disproportionate enlargement and severe lower abdominal pain due to a possible twisted right ovarian mass in pregnancy. Ultrasound played an important role in our initial diagnosis and in raising our suspicion.

We aim to raise awareness of malignant ovarian tumors affecting young females, both outside pregnancy and in this case during pregnancy, the volume of which may rapidly increase within a very short period while the tumor remains asymptomatic. This poses a significant challenge for gynecologists.

Dysgerminomas are mostly unilateral (15\% of dysgerminomas are bilateral), solid, and nodular. They have a smooth, bosselated (knobby) external surface which is soft, fleshy, either cream-colored, gray, pink, or tan when cut. Hemorrhage and necrosis are common but less prominent than in other malignant tumors [11].

Pregnancy associated with ovarian malignancy presents multiple challenges. The ideal treatment for the mother can be hindered at times with the ongoing pregnancy. Furthermore, if conservative treatment and follow-up by imaging is the option, some imaging modalities such as CT of the abdomen and pelvis may pose an increased (yet small) risk to the developing fetus. An additional factor is that the rarity of ovarian cancer with pregnancy means that most evidence does not come from randomized controlled trials and that there will be more dependence on expert opinion, case reports, and retrospective studies in these cases. Nevertheless, the principles of management remain the same, and proper counseling of patients and individualizing treatment on a case-to-case basis is important.

Optimal management starts with proper counseling of all patients undergoing surgery for emergency ovarian torsion. An oophorectomy should be performed if the lesion is highly suspicious for malignancy during the operation. In cases that are suspicious of malignancy before the operation, the surgery should be undertaken by the oncology teams (they were available in our case). Figure 3 shows intraoperative findings of a suspicious right ovary later confirmed by histology to be dysgerminoma.

After confirming malignancy by histopathology, there are various factors to consider: the stage of cancer, patient wishes and preferences, reproductive history, the effects of imaging used for staging or follow-up of cancer on the fetus, gestational age, and whether terminating the pregnancy would be an option to improve survival or morbidity for the mother.

\section{Conclusion}

Patients with ovarian dysgerminoma in pregnancy can have favorable outcomes. Treatment options should be discussed with the patient and tailored on a case-to-case basis. Continuing the pregnancy, leading to viable childbirth, remains an option in some cases, especially if the dysgerminoma is unilateral, without ascites or metastasis, and not involving the peritoneal cavity or other pelvi-abdominal organs. 


\section{Case Reports in Oncology}

Case Rep Oncol 2021;14:141-146

\begin{tabular}{l|c|c|}
\hline DOI: 10.1159/000513622 & (c) 21 The Author(s). Published by S. Karger AG, Basel
\end{tabular} www.karger.com/cro

Youssef et al.: Ovarian Dysgerminoma in Pregnant Women with Viable Fetus

\section{Acknowledgement}

The authors would like to acknowledge the Qatar National Library and co-workers, without whom this work would not be possible.

\section{Statement of Ethics}

The authors have no ethical conflicts to disclose. The work was conducted ethically in accordance with the guideline of the Medical Research Center of Hamad Medical Corporation and after obtaining their approval. Written informed consent was obtained from the patient for publication of this case report and any accompanying images.

\section{Conflict of Interest Statement}

The authors declare that they have no relevant financial interests.

\section{Funding Sources}

Qatar National Library.

\section{Author Contributions}

Dr. R. Youssef and Dr. G.S. Ahmed did the literature review and wrote the manuscript. Dr. S. Alhyassat wrote the histopathology part of the manuscript. Dr. S. Badr, Dr. A. Sabry, and Dr. S. Kohla reviewed and edited the manuscript.

\section{References}

1 Quirk JT, Natarajan N. Ovarian cancer incidence in the United States, 1992-1999. Gynecol Oncol. 2005;97(2): 519-23.

2 Bekaii-Saab T, Einhorn LH, Williams SD. Late relapse of ovarian dysgerminoma: case report and literature review. Gynecol Oncol. 1999 Jan;72(1):111-2.

3 De Palo G, Pilotti S, Kenda R, Ratti E, Musumeci R, Mangioni C, et al. Natural history of dysgerminoma. Am J Obstet Gynecol. 1982 Aug;143(7):799-807.

4 Smith HO, Berwick M, Verschraegen CF, Wiggins C, Lansing L, Muller CY, et al. Incidence and survival rates for female malignant germ cell tumors. Obstet Gynecol. 2006 May;107(5):1075-85.

5 Zanetta G, Bonazzi C, Cantù M, Binidagger S, Locatelli A, Bratina G, et al. Survival and reproductive function after treatment of malignant germ cell ovarian tumors. J Clin Oncol. 2001 Feb;19(4):1015-20.

6 Tangir J, Zelterman D, Ma W, Schwartz PE. Reproductive function after conservative surgery and chemotherapy for malignant germ cell tumors of the ovary. Obstet Gynecol. 2003 Feb;101(2):251-7.

7 Gershenson DM. Update on malignant ovarian germ cell tumors. Cancer. 1993 Feb;71(4 Suppl):1581-90.

8 Chen Y, Luo Y, Han C, Tian W, Yang W, Wang Y, et al. Ovarian dysgerminoma in pregnancy: a case report and literature review. Cancer Biol Ther. 2018 Aug;19(8):649-58.

9 Ueda M, Ueki M. Ovarian tumors associated with pregnancy. Int J Gynaecol Obstet. 1996 Oct;55(1):59-65.

10 Anwar S, Rehan B, Hameed G. MRI for the diagnosis of ultrasonographically indeterminate pelvic masses. J Pak Med Assoc. 2014;64(2):171-4.

11 Gupta M, Saini V. Germ cell tumors and their association with pregnancy. In: Ahmed RG, editor. Germ Cell. IntechOpen; 2017. Available from: https://www.intechopen.com/books/germ-cell/germ-cell-tumors-andtheir-association-with-pregnancy. 